TITLE:

\title{
Molecular basis for intracranial aneurysm formation.
}

$\operatorname{AUTHOR}(\mathrm{S})$ :

Fukuda, Miyuki; Aoki, Tomohiro

CITATION:

Fukuda, Miyuki ...[et al]. Molecular basis for intracranial aneurysm formation.. Acta neurochirurgica Supplement 2015, 120: 13-15

ISSUE DATE:

2015

URL:

http://hdl.handle.net/2433/199650

\section{RIGHT:}

The final publication is available at Springer via http://dx.doi.org/10.1007/978-3-31904981-6_2.; 許諾条件により本文ファイルは2017-01-01に公開.; This is not the published version. Please cite only the published version.; この論文は出版社版でありません。引用 の際には出版社版をご確認ご利用ください。 


\title{
Molecular basis for the intracranial aneurysm formation
}

\author{
Miyuki Fukuda 1, 2, 3 and Tomohiro Aoki 2, 3,4 \\ ${ }^{1}$ Department of Neurosurgery, Kyoto University Graduate School of Medicine, Kyoto, Japan, ${ }^{2}$ \\ Department of Pharmacology, Kyoto University Graduate School of Medicine, Kyoto, Japan, ${ }^{3}$ Core
}

Research for Evolutional Science and Technology (CREST), Kyoto University Graduate School of

Medicine, Kyoto, Japan, ${ }^{4}$ Innovation Center for Immunoregulation Technologies and Drugs (AK

project), Kyoto University Graduate School of Medicine, Kyoto, Japan

Corresponding author;

Tomohiro Aoki

Innovation Center for Immunoregulation Technologies and Drugs (AK project), Kyoto University

Graduate School of Medicine, Konoe-cho Yoshida, Sakyo-ku, Kyoto City, Kyoto, 606-8501, Japan

TEL : +81-75-753-9511 / FAX : +81-75-753-9500

tomoaoki@kuhp.kyoto-u.ac.jp 


\section{Summary}

Intracranial aneurysm (IA) is a socially important disease because of the high prevalence and the

severity of resultant subarachnoid hemorrhage after rupture. The major concern of current IA

treatment is the lack of less invasive medical therapy for many patients with IAs except for surgical

procedures. Current situation is mostly due to the lack of knowledge regarding the regulating

mechanisms of IA formation. Hemodynamic stress, especially high wall shear stress, loaded on the

arterial bifurcation sites is recognized as a trigger of IA formation from studies achieved in the field

of fluid dynamics. As the other point of view, many researches using human specimen have revealed

the presence of active inflammatory responses, such as the infiltration of macrophages, in the

pathogenesis of IA. Based on these findings, recent experimental studies, mainly using animal

models of IA, have revealed some of molecular mechanisms linking hemodynamic stress and

long-lasting inflammation in IA walls. Currently, we propose the notion that IA is a chronic

inflammatory disease regulated by the positive feedback loop consisting of

COX-2-ProstaglandinE 2 -EP2-NF- $\mathrm{B}$ pathway triggered under hemodynamic stress and macrophage

infiltration via NF-кB-mediated MCP-1 induction. These findings provide the future prospection for 
the development of therapeutic drugs for IAs.

\section{Key words}

Intracranial aneurysm, subarachnoid hemorrhage, inflammation, NF-кB, macrophage, prostaglandin,

COX-2, EP2, MCP-1, statin

\section{Text; Findings from studies using human IA specimen}

Recent studies in the field of fluid dynamics have demonstrated the close interactions of hemodynamics with IAs [10]. For example, among various parameters of hemodynamics, high wall shear stress loaded on the arterial bifurcation sites, where IA is formed, is associated with IA formation and growth [10]. High wall shear stress can, therefore, be recognized as a trigger of IA formation.

On the other point of view, in the field of histopathological analyses, gene linkage analyses and comprehensive gene expression analyses, it have been revealed that active inflammatory responses such as macrophage infiltration and the expression of various cytokines are present in human IAs [8].

For example, Shi et al. analyzed gene expression profiles in human IA lesions by a microarray technique and revealed that inflammation-related biological pathways, inflammatory response and 
apoptosis, were associated with IA development [11]. Consistent with this, they also confirmed the upregulation of pro-inflammatory genes in human IA walls including interleukin-1 beta (IL-1 $\beta$ ), tumor necrosis factor alpha (TNF- $\alpha$ ), vascular cell adhesion molecule 1 (VCAM-1), C-X-C chemokine receptor type 4 (CXCR4) and chemokine ligand 5 (CCL5) [11].

However, studies using human IA specimen, to solve the mechanisms underlying IA formation and development, have the considerable limitations such as the individual heterogeneity of genetic backgrounds and the difficulty of pathological analyses at each period of IA formation from same patients. We, therefore, have developed experimental models of IA to overcome this situation.

Molecular mechanisms regulating IA formation through linking hemodynamic stress and

\section{long-lasting inflammation}

We have established experimental models of IAs by increase of hemodynamics at the bifurcation sites of cerebral arteries through the ligation of carotid artery and salt overloading [5].

Because experimental IA and human IA share histological similarities characterized by the degeneration of arterial wall, the disruption of internal elastic lamina and the infiltration of inflammatory cells, these animal models are suitable for the analyses of the pathogenesis of IAs. 
Indeed, based on recent experimental studies using these models, our understanding of the mechanisms regulating IA formation and development has been remarkably accelerated.

Through the studies using animal models, we have identified nuclear factor kappa B (NF-кB) as a critical transcription factor for IA formation $[1,8]$. NF- $\kappa B$ leads the induction of various pro-inflammatory genes such as monocyte chemoattractant protein-1 (MCP-1), a factor recruiting macrophages in IA walls [7, 8]. Macrophages recruited in cerebral arterial walls by NF-кB-mediated MCP-1 induction, then, produce a large amount of cytokines and proteinases and exacerbate the inflammation associated with IA formation and growth [7, 8]. Here, however, how high wall shear stress induces NF-кB-mediated inflammation and how the inflammation becomes chronic remain to be elucidated.

We have recently demonstrated that the positive feedback loop consisting of cyclooxygenase-2 $(\mathrm{COX}-2)$ - prostaglandin $\mathrm{E}_{2}\left(\mathrm{PGE}_{2}\right)$ - prostaglandin $\mathrm{E}$ receptor 2 (EP2) - NF-אB signaling pathway is formed under high wall shear stress and induces a long-lasting (chronic) inflammation in IA walls $[6,7]$. As previously discussed, at the sites of IA formation, mostly arterial bifurcations, high wall shear stress is loaded and recognized as a trigger of IA formation [7]. In vitro study, using primary 
culture of endothelial cells from human carotid artery, demonstrated the induction of COX-2, prostaglandin-producing enzyme, and its receptor EP2 under high wall shear stress. Consistently, both COX-2 and EP2 expression were also up-regulated in experimental IAs during IA formation and their expression was well co-localized in endothelial cells where wall shear stress was loaded. Here, because either the administration of Celecoxib (a selective COX-2 inhibitor) or EP2 deficiency significantly suppressed both IA formation and inflammatory responses in IA walls such as NF-kB activation and macrophage infiltration, shear stress-activated prostaglandin pathway is identified as a mediator of NF-kB-induced inflammation during IA formation. Indeed, in endothelial cells, treatment with $\mathrm{PGE}_{2}$ or a selective EP2 agonist activated NF-кB and its target, MCP-1. Importantly, COX-2 inhibition suppressed EP2 expression, and vice versa. Thus, once hemodynamic stress induces COX-2 expression in endothelial cells at the bifurcation sites of cerebral arteries, the positive feedback loop consisting of COX-2 - $\mathrm{PGE}_{2}-\mathrm{EP} 2$ - NF-кB was formed resulting in the amplification and the chronicity of inflammation.

\section{Future prospection for the development of therapeutic drugs for IA}

From the recent experimental achievements, NF- $\mathrm{B}$ is recognized as a potential therapeutic 
target for IA treatment [4]. Furthermore, the significant suppression of IA formation and growth in

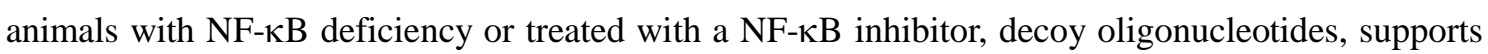
this notion [1].

Statins (3-hydroxy-3 methylglutaryl coenzyme A reductase inhibitors) are originally developed as therapeutic drugs for lipid metabolic abnormality. In addition, statins are well-recognized to have powerful anti-inflammatory and especially anti-NF- $\mathrm{B}$ effects known as a pleiotropic effect of statins. Encouraging by this pleiotropic effect of statins, we administered Pitavastatin, one of statins, to our rat model of IA and demonstrated that Pitavastatin treatment effectively prevented the growth of IAs in rats [3]. Pitavastatin treatment remarkably suppressed the inflammatory responses in IA walls, characterized by NF- $\mathrm{KB}$ activation and subsequent induction of the expression of

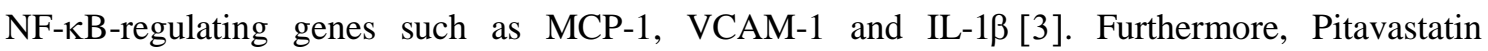
treatment effectively inhibited the degenerative change of IA walls suggesting the preventive effect of Pitavastatin against the rupture of IAs [3]. Here, other kind of statins, Simvastatin and Pravastatin, could also successfully prevented IA growth through the inhibition of inflammation in IA walls, suggesting that statins are potential therapeutic drugs for IAs [2, 9]. 
Based on these findings from experimental animals, we have examined the preventive effect of statins for the rupture of human IAs in Case-Control study in Japan. As a result, we clarified the inverse relationship between the usage of statins and the occurrence of aneurysmal subarachnoid hemorrhage in Japanese population. In detail, statins were administered in $9.4 \%$ of cases with ruptured IAs and $26.0 \%$ of cases with unruptured IAs. The usage of statins, therefore, significantly prevented the rupture of pre-existing IAs with the relative odds ratio of 0.3 [12].

These studies suggest the potential of statins as therapeutic drugs to prevent the growth and rupture of IAs.

\section{Acknowledgement}

Authors would like to express our gratitude to all the researchers, collaborators, technical assistants and secretaries contributing to our studies cited in the present manuscript. Authors also express our sincere gratitude to grants supporting our research works.

\section{References}

[1] Aoki T, Kataoka H, Shimamura M, Nakagami H, Wakayama K, Moriwaki T, Ishibashi R, Nozaki K, Morishita R, Hashimoto N (2007) NF-kB is a key mediator of cerebral aneurysm formation. Circulation 116:2830-40 
[2] Aoki T, Kataoka H, Ishibashi R, Nozaki K, Hashimoto N (2008) Simvastatin suppresses the progression of experimentally induced cerebral aneurysms in rats. Stroke 39:1276-1285

[3] Aoki T, Kataoka H, Ishibashi R, Nakagami H, Nozaki K, Morishita R, Hashimoto N (2009) Pitavastatin suppresses formation and progression of cerebral aneurysms through inhibition of the nuclear factor kappaB pathway. Neurosurgery 64:357-365

[4] Aoki T, Nishimura M (2010) Targeting chronic inflammation in cerebral aneurysms focusing on NFkB as a putative target of medical therapy. Expert Opinion on therapeutic Targets 14:265-273

[5] Aoki T, Nishimura M (2011) The development and the use of experimental animal models to study the underlying mechanisms of CA formation. J Biomed Biotechnol (DOI: $10.1155 / 2011 / 535921)$

[6] Aoki T, Nishimura M, Matsuoka T, Yamamoto K, Furuyashiki T, Kataoka H, Kitaoka S, Ishibashi R, Ishibazawa A, Miyamoto S, Morishita R, Ando J, Hashimoto N, Nozaki K, Narumiya S (2011) $\mathrm{PGE}_{2}$-EP2 signaling in endothelium is activated by haemodynamic stress and induces cerebral aneurysm through an amplifying loop via NF-кB. Br J Pharmacol 163:1237-1249

[7] Aoki T, Narumiya S (2012) Prostaglandins and chronic inflammation.Trends Pharmacol Sci 33:304-11

[8] Kataoka H, Aoki T (2010) Molecular basis for the development of intracranial aneurysm. Expert Rev Neurother 10:173-187

[9] Kimura N, Shimizu H, Eldawoody H, Nakayama T, Saito A, Tominaga T, Takahashi A (2010) 
Effect of olmesartan and pravastatin on experimental cerebral aneurysms in rats. Brain Res $1322: 144-52$

[10] Meng H, Tutino VM, Xiang J, Siddiqui A (2013) High WSS or Low WSS? Complex Interactions of Hemodynamics with Intracranial Aneurysm Initiation, Growth, and Rupture: Toward a Unifying Hypothesis. Am J Neuroradiol (DOI: 10.3174/ajnr.A3558)

[11] Shi C, Awad IA, Jafari N, Lin S, Du P, Hage ZA, Shenkar R, Getch CC, Bredel M, Batjer HH, Bendok BR (2009) Genomics of human intracranial aneurysm wall. Stroke 40:1252-61

[12] Yoshimura Y, Murakami Y, Saitoh M, Yokoi T, Aoki T, Miura K, Ueshima H, Nozaki K; SSS Research Group (2013) Statin Use and Risk of Cerebral Aneurysm Rupture: A Hospital-Based Case-Control Study in Japan. J Stroke Cerebrovasc Dis (DOI: 10.1016/ j.jstrokecerebrovasdis.2013.04.022.)

\section{Figure Legend}

Schema to demonstrate our hypothesis for the potential mechanisms underlying the chronicity of inflammation contributing to intracranial aneurysm formation.

Note the presence of positive feedback loop consisting of $\mathrm{PGE}_{2}-\mathrm{NF}-\kappa \mathrm{B}$ signaling under hemodynamic stress and macrophage infiltration via NF-кB-mediated MCP-1 induction.

\section{Conflict of interest statement}

The authors declare that they have no conflict of interest. 